\title{
Interface magnetism studied by optical second harmonic generation
}

\author{
G. Spierings, V. Koutsos, H.A. Wierenga, M.W.J. Prins, D. Abraham and Th. Rasing \\ Research Institute for Materials, University of Nijmegen, Toernooiveld, 6525 ED Nijmegen, The Netherlands
}

\begin{abstract}
Optical second harmonic generation studies of $\mathrm{Co} / \mathrm{Au}$ interfaces show a clear magnetic field induced effect at these interfaces, leading the way to a nonlinear optical probe that is uniquely sensitive to surface and interface magnetism.
\end{abstract}

\section{Introduction}

The magnetism of thin films and of magnetic surfaces and interfaces is a topic of great current interest, from both fundamental and practical points of view [1,2]. Novel epitaxially grown magnetic structures not only hold promise for new devices but also for new physics as magnetic phases can be grown that do not occur naturally [3-5]. To improve our understanding of magnetic properties at an interface we have undertaken the development of a new technique: magnetic optical second harmonic generation (MSHG). SHG derives its surface sensitivity from the symmetry breaking at an interface. On theoretical grounds it can be estimated that SHG should also be sensitive to magnetic order at an interface and recently a magnetic component to the optical nonlinear response at the $\mathrm{Fe}$ /vacuum interface has been demonstrated [6]. Different from a linear optical technique like the Kerr effect which is bulk sensitive, MSHG may lead to a novel probe for interface magnetism. As a feasibility test for this technique we have conducted studies utilizing buried magnetic films where the interfaces are optically accessible. These experiments have clearly revealed a strongly magnetization dependent SHG signal from buried $\mathrm{Co} / \mathrm{Au}$ interfaces with relative susceptibility comparable to that at $\mathrm{Fe} /$ vacuum and with signal/noise sufficient to observe magnetic hysteresis. The present and fu-

Correspondence to: Dr. Th. Rasing, Research Institute for Materials, University of Nijmegen, Toernooiveld, NL-6525 ED Nijmegen, The Netherlands. Tel. 080-653102/652121; fax 080-553450; e-mail: theoras@sci.kun.nl. ture work is aimed at detecting possible bulk contributions arising from interference with either linear or higher order (bulk allowed) nonlinear magneto-optic responses.

\section{Theory}

SHG arises from the nonlinear polarization $\boldsymbol{P}(2 \omega)$ induced by an incident laser field $E(\omega)$. The surface sensitivity of SHG for cubic materials is a result of breaking the cubic symmetry at a surface or interface, in that way allowing for a nonvanishing dipole contribution from the surface $[7,8]$ :

$P(2 \omega)=\boldsymbol{X}_{\mathrm{d}}^{(2)} \boldsymbol{E}(\omega) \boldsymbol{E}(\omega)$,

where $\boldsymbol{X}_{\mathrm{d}}^{(2)}$ is a third rank tensor that is identically zero in the bulk for media with inversion symmetry. The presence of a magnetization $M$ does not break the inversion symmetry, because $\boldsymbol{M}$ is an axial vector with even parity under inversion. However, $\boldsymbol{M}$ does lower the symmetry of both bulk and surface, which means that it induces extra nonzero elements [9]. For our experiment, the nonzero magnetic contributions that are odd in $\boldsymbol{M}$ are the most relevant, as they change sign upon reversal of the direction of the magnetization, giving rise to a different SHG intensity. This we can write as

$\boldsymbol{P}(2 \omega) \sim\left(\boldsymbol{X}_{\mathrm{NM}} \pm \boldsymbol{X}_{\mathrm{M}}\right) \boldsymbol{E}(\omega) \boldsymbol{E}(\omega)$

where $\boldsymbol{X}_{\mathrm{NM}}$ indicates the term even or independent on $\boldsymbol{M}$, and $\boldsymbol{X}_{\mathbf{M}}$ is the odd magnetic contribution. 


\section{Experiment}

For the experiment we used the frequency doubled output at $532 \mathrm{~nm}$ of a Q-switched NdYAG laser. The laser beam was incident at an angle of $45^{\circ}$ on the samples, which were mounted in between the poles of an electromagnet. The magnetization could be switched in the vertical direction (up or down) and the polarization of the incident and detected SHG light was in the (horizontal) plane of incidence (p-polarized), see fig. 1. The samples were thin films of $\mathrm{Co}$ and $\mathrm{Au}$, evaporated at about $2 \AA / s$ at a pressure of $5 \times$ $10^{-7}$ Torr and the substrates at room temperature. The substrates were glass or fused quartz, cleaned with ethanol and blow dried with nitrogen. Three samples were studies: $\mathrm{Co}-\mathrm{Au}, \mathrm{Au}-$ $\mathrm{Co}-\mathrm{Au}$, and $\mathrm{Co}-\mathrm{Au}-\mathrm{Co}-\mathrm{Au}$. This means we have samples with 1,2 and $3 \mathrm{Co}-\mathrm{Au}$ interfaces, respectively.

\section{Results and discussion}

Fig. 2a shows the observed MSHG for sample 1, a $500 \AA$ Co film with $50 \AA$ Au on top. We see an increase of the SHG intensity for $M$ down and a decrease for $M$ up. On the other hand in sample 2, an Au-Co-Au film (1000-50-50 ̊) we could not detect a clear difference between $\boldsymbol{M}$ up and down (see fig. 2b). Finally, for sample 3, a $\mathrm{Co}-\mathrm{Au}-\mathrm{Co}-\mathrm{Au}$ film (500-50-50-50 ̊), a magnetization dependent signal was observed (see fig. $2 c)$. The observed intensity changes can be related to the magnitude and phases of the mag-

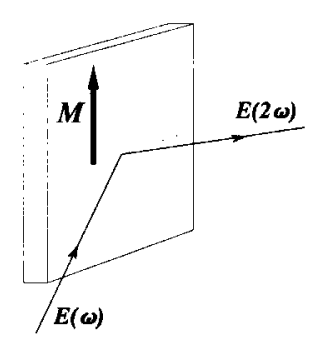

Fig. 1. Schematic representation of experimental conditions.

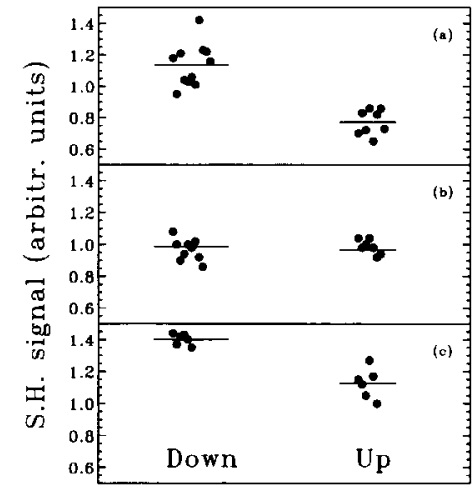

Fig. 2. Magnetic field induced second harmonic generation signal of (a) a magnetized $500 \AA$ Co film with a $50 \AA$ Au layer on top; (b) a magnetized multilayer of $1000 \AA \mathrm{Au}-50 \AA \mathrm{Co}-50$ $\AA \mathrm{Au}$; (c) a magnetized multilayer film of $500 \AA \mathrm{A} \mathrm{Co}-50 \AA$ $\mathrm{Au}-50 \AA \mathrm{Co}-50 \AA \mathrm{Au}$. Light is incident from the Au side at an angle of $45^{\circ}$.

netic and nonmagnetic tensor elements:

$$
\begin{aligned}
\frac{I_{\uparrow}-I_{\downarrow}}{I_{\uparrow}+I_{\downarrow}} & =\frac{\left|\boldsymbol{X}_{\mathrm{NM}}+\boldsymbol{x}_{\mathrm{M}}\right|^{2}-\left|\boldsymbol{x}_{\mathrm{NM}}-\boldsymbol{x}_{\mathrm{M}}\right|^{2}}{\left|\boldsymbol{X}_{\mathrm{NM}}+\boldsymbol{X}_{\mathrm{M}}\right|^{2}+\left|\boldsymbol{x}_{\mathrm{NM}}-\boldsymbol{X}_{\mathrm{M}}\right|^{2}} \\
& =\frac{2 \operatorname{Re}\left\{\boldsymbol{X}_{\mathrm{NM}}^{*} \boldsymbol{X}_{\mathrm{M}}\right\}}{\left|\boldsymbol{X}_{\mathrm{NM}}\right|^{2}-\left|\boldsymbol{X}_{\mathrm{M}}\right|^{2}} .
\end{aligned}
$$

So the direction of the change of the total SHG signal depends on the relative phases of $\boldsymbol{X}_{\mathrm{M}}$ and $\boldsymbol{X}_{\mathrm{NM}}$. For sample 1, the results look similar to that observed by Reif et al. [6] on the Fe(110) surface. Taking the direction of the light beams into account, our results are exactly opposite, as the phase of the $\boldsymbol{X}_{\mathrm{M}}$ component is related to the relative orientation of the wave vector of the incident e.m. field and the magnetization. However, keeping in mind that the measured SHG signal contains contributions from the magnetic $\mathrm{Co} / \mathrm{Au}$ interface as well as from the nonmagnetic $\mathrm{Au} /$ air interface, this can easily be explained, because as shown in eq. (3), the sign of the change of the MSHG signal will be totally determined by the relative phases of these different $X$ components. The results of sample 2 are very interesting because they indicate that the two different $\mathrm{Co} / \mathrm{Au}$ interfaces tend to cancel each other. From an experimental point of view, the 
incident laser beam first sees an $\mathrm{Au}-\mathrm{Co}$ and then a $\mathrm{Co}-\mathrm{Au}$ interface. Based on symmetry considerations one expects the $X$-components to be exactly out of phase, thus the net effect will be zero. This observation is supported by the data on 3 $\mathrm{Co} / \mathrm{Au}$ interfaces, where a magnetic effect reappears. (see fig. 2c).

It should be stressed at this point that the interface sensitivity of SHG is based on the breaking of the macroscopic inversion symmetry at interfaces, and does not depend on the detailed interface structure. The latter does of course affect the magnitude of the different allowed X-components. For the studied $\mathrm{Co} / \mathrm{Au}$ system, both metal films individually will have an average inversion symmetry, whether they consist of large single domains or small microcrystallites. Based on the results of Reif et al. [6] and on earlier SHG work on metal surfaces like Ni [10], which also proved the extreme surface sensitivity of SHG, our observations can most likely be ascribed to a magnetization induced contribution to the SHG from the Co/Au interface.

In conclusion, we have introduced a novel technique to study interface magnetism by magnetic induced optical second harmonic generation. Different from the linear magneto-optical (KERR) effect, MSHG may be really surface and interface specific, which opens up interesting pos- sibilities for the study of interface magnetism for multilayer systems. Future investigations are planned to explore these possibilities in more detail and to get a better quantitative understanding of the observed results.

Part of this work was supported by the stichting Fundamenteel Onderzoek der Materie (FOM), which is financially supported by the Nederlandse Organisatie voor Wetenschappelijk Onderzoek (NWO).

\section{References}

[1] L.M. Falicov et al., J. Mater. Res. 5 (1990) 1299.

[2] J. Mathon, Rep. Prog. Phys. 51 (1988) 1.

[3] M. Stampanoni, A. Vaterlaus, M. Aeschlimann, F. Meier and D. Pescia, J. Appl. Phys. 64 (1988) 5321.

[4] W. Durr, M. Taborelli, O. Paul, R. Germar, W. Gudat, D. Pescia and M. Landholt, Phys. Rev. Lett. 62 (1989) 206.

[5] S.T. Purcell, W. Folkerts, M.T. Johnson, N.W.E. McCee, K. Jager, J. aan de Stegge, W.B. Zeper and W. Hoving, Phys. Rev. Lett. 67 (1991) 903.

[6] J. Reif, J. C. Zink, C.M. Schneider and J. Kirschner, Phys. Rev. Lett. 67 (1991) 2278.

[7] Y.R. Shen, The Principle of Nonlinear Optics (Wiley, New York, 1984); J. Vac. Sci. Technol. B 3 (1985) 1464.

[8] G.L. Richmond, J.M. Robinson and V.L. Shannon, Prog. Surf. Sci. 28 (1988) 1.

[9] R. Pan and Y.R. Shen, Phys. Rev. B 39 (1989) 1229.

[10] X.D. Zhu, Th. Rasing and Y.R. Shen, Phys. Rev. Lett. 61 (1988) 2883. 